\title{
Avaliação in vitro das atividades antiinflamatória, antioxidante e antimicrobiana do alcalóide montanina
}

\author{
Tatiana S. Castilhos ${ }^{1}$, Raquel B. Giordani ${ }^{1}$, Amélia T. Henriques ${ }^{1}$, Fábio S. Menezes $^{2}$, \\ José Ângelo S. Zuanazzi ${ }^{1 *}$ \\ ${ }^{1}$ Programa de Pós-Graduação em Ciências Farmacêuticas, Faculdade de Farmácia, Universidade Federal do Rio \\ Grande do Sul, Av. Ipiranga, 2752, 90610-000, Porto Alegre, RS, Brasil, \\ ${ }^{2}$ Departamento de Produtos Naturais e Alimentos, Faculdade de Farmácia, Universidade Federal do Rio de \\ Janeiro, BI, Ilha do Fundão, 21941-590, Rio de Janeiro, RJ, Brasil
}

\begin{abstract}
RESUMO: Diversas propriedades biológicas, dentre as quais atividade antiparasitária, antitumoral, antiviral e anticolinesterásica são associadas aos extratos e produtos isolados, incluindo alcalóides de espécies de Amarilidáceas. Neste trabalho verificou-se para o alcalóide montanina, isolado dos bulbos de Rhodophiala bifida (Herb.) Traub (Amaryllidaceae), atividade antioxidante e ação inibitória sobre o crescimento de culturas de Saccharomyces cerevisae (ATCC 2601), Pseudomonas aeruginosa (ATCC 27853), Staphylococcus aureus (ATCC 6538), Staphylococcus epidermides (ATCC 12228) e Escherichia coli (ATCC 25922). A atividade antiinflamatória avaliada através da determinação da capacidade antiquimiotáxica da amostra apresentou resultados não significativos na dose empregada.
\end{abstract}

Unitermos: Amaryllidaceae, Rhodophiala bifida, montanina, alcalóides.

\begin{abstract}
In vitro evaluation of the antioxidant, anti-inflammatory and antimicrobial activities of the montanine alkaloid". Several biological properties, such as antiparasite, antitumor, antiviral and anticholinesterasic activities, are associated with extracts and isolated products, including alkaloids from Amaryllidaceae species. In this study, the antioxidant activity and inhibitory action on the growth of cell cultures of Saccharomyces cerevisae (ATCC 2601), Pseudomonas aeruginosa (ATCC 27853), Staphylococcus aureus (ATCC 6538), Staphylococcus epidermides (ATCC 12228) and Escherichia coli (ATCC 25922) were observed to the montanine alkaloid, isolated from the bulbs of Rhodophiala bifida (Herb.) Traub (Amaryllidaceae). The antiinflammatory activity, which was evaluated through the sample antichemotaxis ability determination, was not significant in the used dose.
\end{abstract}

Keywords: Amaryllidaceae, Rhodophiala bifida, montanine, alkaloids.

\section{INTRODUÇÃO}

As propriedades terapêuticas de plantas da família das Amarilidáceas são conhecidas desde a antigüidade, quando exemplares de Narcissus poeticus L. eram utilizados pelo médico grego Hipócrates no tratamento de tumores uterinos (Gabrielsen et al., 1992). Em dias atuais, em países dos continentes europeu e africano, espécies desta família ainda são empregadas na medicina tradicional sendo indicadas como agentes eméticos, purgativos e antiparasitários, assim como no tratamento de doenças urinárias, venéreas, gastrintestinais, respiratórias, febres, entre outras (Shale et al., 1999; Louw et al., 2002; Agra et al., 2007).

Em estudos preliminares da composição química dos diferentes órgãos vegetais de espécies desta família, verificou-se uma grande quantidade de compostos nitrogenados, dentre os quais, alcalóides estruturalmente diferenciados, não encontrados em outras famílias vegetais (Louw et al., 2002). Atividades biológicas e farmacológicas marcantes têm sido atribuídas aos alcalóides de Amarilidáceas, tais como atividade anticolinesterásica da galantamina, citotoxicidade e ação antitumoral da licorina, além de atividades antiinflamatória, antiviral e antimalárica relatadas de muitos núcleos desses alcalóides. (Harvey, 1995; Weniger et al., 1995; Rhee et al., 2001; Sener et al., 2003; BarbosaFilho et al., 2006a,b).

Em continuidade à nossa investigação sistemática de estudo químico e potencial farmacológico das espécies de Amarilidáceas de ocorrência no Rio Grande do Sul (Hofmann Jr et al., 2003; Hofmann Jr et al., 2004; Sebben, 2005; Silva, 2005) o presente estudo envolveu a avaliação biológica in vitro das atividades antiinflamatória, antimicrobiana e antioxidante do alcalóide montanina (Figura 1), substância abundante nos bulbos de Rhodophiala bifida (Herb.) Traub. 


\section{MATERIAL E MÉTODOS}

\section{Amostra}

O extrato etanólico dos bulbos frescos de $R$. bifida foi submetido à extração ácido-base obtendo-se uma fração diclorometano enriquecida de alcalóides. Essa fração foi submetida à Cromatografia Líquida a Vácuo (CLV) que ao ser eluída com diclorometano gerou o alcalóide isolado montanina (Figura 1) (Castilhos, 2004). Uma exsicata do vegetal foi depositada no Herbário da Universidade Estadual de Campinas, com o número 13.6352. Para a identificação e determinação de pureza do produto foram utilizadas técnicas espectroscópicas (RMN ${ }^{1} \mathrm{H}$ e $\mathrm{RMN}{ }^{13} \mathrm{C}$ ), além de Cromatografia em Camada Delgada (CCD) e Cromatografia Líquida de Alta Eficiência (CLAE), em análises frente à amostra de referência conforme descrito por Castilhos (2004).

\section{Avaliação da atividade antiinflamatória}

Para avaliação da atividade antiinflamatória foi empregado o modelo de quimiotaxia, investigado através do método da câmara de Boyden (Boyden, 1962), conforme descrito por Suyenaga (2002). Nesta técnica, o potencial antiinflamatório da montanina (100 $\mu \mathrm{g} / \mathrm{mL}$ ) pôde ser avaliado por meio da sua influência inibitória sobre a motilidade dos leucócitos em suspensão (compartimento superior) frente a um agente quimiotáxico (compartimento inferior), determinada pelo espaço percorrido pelo leucócito através do filtro de policarbonato colocado entre os compartimentos da câmara. Microscópio óptico (Nikon modelo Alpha-Phot2 YS2-H.A), com ampliação de 40 vezes, foi utilizado na leitura dos filtros e esta foi desenvolvida com o foco inicialmente ajustado no plano superior do filtro, sendo este plano de observação lentamente aprofundado até que apenas duas células fossem visíveis em foco. A leitura foi realizada em cinco campos de cada filtro e a capacidade migratória dos leucócitos foi avaliada de acordo com a distância, em micrômetros, medida entre o plano superior do filtro e o plano final de observação (Zigmond; Hirsch, 1973). Os resultados expressaram a média das distâncias encontradas nas leituras. Os resultados foram expressos como média +/- erro padrão da média (epm), analisados pelo teste $t$ de Student e comparados com a média das distâncias determinadas para células-controle não tratadas (Snedecor; Cochran, 1974). A diferença foi considerada estatisticamente significativa quando $\mathrm{p} \leq 0,05$.

\section{Avaliação da atividade antimicrobiana}

A atividade antibacteriana e antifúngica da montanina foi avaliada de acordo com o método de bioautografia por imersão modificado (Rios et al., 1988). Para este teste foram utilizados os seguintes microrganismos: Micrococcus luteus (ATCC 9341),
Pseudomonas aeruginosa (ATCC27853), Staphylococcus aureus (ATCC 6538), Staphylococcus epidermides (ATCC 12228), Escherichia coli (ATCC 25922), Candida albicans (ATCC 10231) e Saccharomyces cerevisae (ATCC 2601), na concentração de $10^{8} \mathrm{UFC} / \mathrm{mL}$, sendo utilizados $5 \mathrm{~mL}$ de caldo para a suspensão. Deve-se ressaltar que $10^{8} \mathrm{UFC} / \mathrm{mL}$ correspondem à metade da concentração de microorganismos segundo o tubo 1 da escala de MacFarland e ainda, que os meios de cultura utilizados no estudo são descritos por Grove e Randall (1955) e adquiridos comercialmente. Primeiramente, para as bactérias, as culturas foram repicadas para tubos inclinados contendo meio de cultura número 1 de GroveRandall e estes foram incubados a $37^{\circ} \mathrm{C}$ durante 24 horas. A partir destas novas culturas, foram realizados repiques incubados em meio nutritivo número 3 de Grove-Randall, por mais 24 horas a $37^{\circ} \mathrm{C}$. Após este período, esta suspensão de bactérias foi adicionada na proporção de $1 \%(\mathrm{v} / \mathrm{v})$ a frascos erlenmeyer contendo meio de cultura número 11 de Grove-Randall previamente liquefeito, obtendo-se assim os inóculos a serem utilizados. Para os fungos, as suspensões de leveduras foram preparadas a partir de repiques das culturas mantidas sob refrigeração, para $5 \mathrm{~mL}$ de caldo Sabouraud, enquanto na preparação dos inóculos, também preparado na proporção de $1 \%$ em relação às suspensões adicionadas, empregou-se agar Sabouraud liquefeito. Placas de Petri estéreis (20 mm de altura x $100 \mathrm{~mm}$ de diâmetro) foram utilizadas para a realização do ensaio. Nestas, foram depositadas cromatoplacas de gel de sílica GF ${ }_{254}$ (50 mm de altura x 70 mm de largura) onde a solução-teste (montanina; 1 $\mathrm{mg} / \mathrm{mL}$ ) foi aplicada, em pontos distintos, em alíquotas de 5, 10, 15, 20 e $50 \mu \mathrm{L}$. Soro fisiológico e clorofórmio marca Sigma, foram utilizados como branco e controle de solvente, respectivamente, aplicados em volume de $5 \mu \mathrm{L}$, da mesma forma que as substâncias de referência preparadas a uma concentração de $1 \mathrm{mg} / \mathrm{mL}$ : cloranfenicol (bactérias) e cetoconazol (fungos). As placas foram incubadas durante um período 24 horas a $37{ }^{\circ} \mathrm{C}$ e logo após, para melhor visualização das zonas de inibição do crescimento microbiano, sofreram nebulização com solução aquosa de 2,3,5-trifenil-cloreto de tetrazólio (20 $\mathrm{mg} / \mathrm{mL}$ ) sendo incubadas por mais 1 hora (Hamburger; Cordel 1967; Hamburger; Hostettman, 1991). Os testes foram realizados em triplicata para cada microrganismo e os resultados basearam-se exclusivamente na formação ou não de halos de inibição, caracterizando o caráter qualitativo do método.

\section{Avaliação da atividade antioxidante}

A atividade antioxidante da montanina foi determinada através do método de doseamento fotocolorimétrico do radical 2,2-difenil-1-picrilhidrazilhidrato (DPPH), em processo guiado pela redução e descoloração deste em solução alcoólica na presença de agentes antioxidantes, conforme a técnica descrita por 
Mensor et al. (2001) sendo utilizada como substância controle, padrão de rutina (Merck) a $1 \mathrm{mg} / \mathrm{mL}$. A amostra adicionada de $1 \mathrm{~mL}$ de etanol foi utilizada como branco e a solução de DPPH (1 mL) também adicionada de etanol $(2,5 \mathrm{~mL})$ serviu de controle negativo. $\mathrm{O}$ controle positivo foi submetido às mesmas condições de preparação e análise utilizadas para a amostra. Após uma reação de 30 min à temperatura ambiente, a absorvância (ABS) foi determinada a $518 \mathrm{~nm}$ em espectrofotômetro Shimadzu UV-2200 e o percentual de atividade antioxidante obtido através da fórmula abaixo:

$$
\mathrm{AA} \%=100-\left\{\left[\left(\mathrm{ABS}_{\text {amostra }}-\mathrm{ABS}_{\text {branco }}\right) \times 100\right] / \mathrm{ABS}_{\text {controle }}\right\}
$$

Os testes foram feitos em triplicata e os resultados representados em média +/- desvio padrão. O teste $t$ de Student foi utilizado para a comparação entre duas médias obtidas e a variância entre duas ou mais médias foi determinada através da análise da variância (ANOVA). A diferença foi considerada estatisticamente significativa quando $\rho \leq 0,05$.

\section{RESULTADOS}

Os resultados do tratamento prévio e da influência do alcalóide montanina sobre a locomoção dos neutrófilos em suspensão estão apresentados na Tabela 1 , onde são comparados com os valores obtidos para as células-controle.

Considerando-se a distância migrada (em $\mu \mathrm{M})$ pelas células-controle não tratadas como valor equivalente a 100\% de migração celular, foi possível determinar para a suspensão de leucócitos tratados com montanina (100 $\mu \mathrm{g} / \mathrm{mL}$ ), valores muito próximos àqueles apresentados pelo grupo controle, caracterizando a incapacidade da amostra, na dose utilizada, em impedir a migração leucocitária, uma das etapas do processo inflamatório.

Durante a avaliação da atividade antimicrobiana, somente $M$. luteus (ATCC 9341) e $C$. albicans (ATCC 10231) foram resistentes à adição de montanina em todas as quantidades testadas. Para os demais microrganismos, apesar da natureza qualitativa do teste, a aplicação da amostra em alíquotas de concentrações crescentes possibilitou que os valores de concentração inibitória mínima sobre cada microrganismo pudessem ser estimados, sendo apresentados na Tabela 2. A atividade antimicrobiana não foi observada para o solvente utilizado na solubilização da amostra.

Apesar de um percentual de atividade antioxidante médio determinado em cerca de 36\% para montanina a $3,5 \mu \mathrm{g} / \mathrm{mL}$, a amostra não apresentou comportamento linear no que se refere ao acréscimo dessa atividade quando testada em concentrações crescentes. Conforme o Gráfico 1, valores negativos de percentual de atividade foram observados para montanina a 17,5 e 35 $\mu \mathrm{g} / \mathrm{mL}$, enquanto percentuais de atividade antioxidante média de 0,7 e 3,6\%, puderam ainda ser observados para o produto a 87,5 e $175 \mu \mathrm{g} / \mathrm{mL}$, respectivamente, porém estes não foram considerados significativos.

\section{DISCUSSÃO}

O gênero Rodophiala contempla cerca de 30 espécies já relatadas, (Traub, 1953; Dahlgren, 1985; Meerow et al., 1998), entretanto, no estado do Rio Grande do Sul exemplares desse gênero nunca haviam sido relatados anteriormente. Ainda, deve-se salientar que além de $R$. bifida (Herb) Traub ser a única espécie representante deste gênero no estado, é também a única que teve seu conteúdo químico já investigado (Wildmann; Brow, 1968; Cordell et al., 1989). A presença da montanina como produto majoritário nos bulbos de $R$. bifida já havia sido relatada por Wildmann \& Brow (1968) em estudo onde também foi evidenciado o isolamento de pancracina, outro alcalóide de mesmo núcleo fundamental. Mais recentemente, em trabalho desenvolvido por nosso grupo de pesquisa (Castilhos, 2004), detectou-se a presença de nangustina em extratos de $R$. bifida, confirmando a predominância de alcalóides de um mesmo tipo de núcleo como produtos da biossíntese dessa espécie.

Labraña et al. (2002) verificaram para pancracina fraca atividade antiparasitária sobre Trypanosoma brucei rhodesiense, Trypanosoma cruzi e Plasmodium falciparum, enquanto nangustina foi considerado inativo frente a esses microrganismos. Dentre estes alcalóides de núcleo idêntico ao observado para montanina, somente para nangustina e pancracina são encontrados dados referentes a avaliações biológicas.

Conforme Cassels et al. (1995) a presença de um sistema difenólico orto-substituído por dois grupamentos metoxila está intimamente associada à ação antioxidante da boldina, enquanto que em apomorfina e outros alcalóides benziltetraisoquinolínicos, associa-se tal atividade à ocorrência de núcleo catecol (Martinez et al., 1992), características ausentes na estrutura de montanina. Além disso, também a atividade antiinflamatória parece ser influenciada pela presença de grupamentos hidroxila (Harborne; Williams, 2000) e metoxila, estes últimos encontrados em significativo número na estrutura da colchicina, antiinflamatório natural cuja ação deve-se principalmente à diminuição da motilidade e adesividade dos neutrófilos envolvidos no processo inflamatório (Bruneton, 2001). Neste estudo preliminar sobre as potencialidades farmacológicas da montanina, não foi constatada atividade antiinflamatória e verificada atividade antioxidante moderada (36\%) a 3,5 $\mu \mathrm{g} / \mathrm{mL}$, podendo ter relação com características estruturais do 
alcalóide como, por exemplo, a ausência de determinados substituintes-chaves, essenciais para o desencadeamento da atividade.

Da mesma forma observa-se, nos ensaios in vitro, a ausência de muitos processos metabólicos que, em teste realizado in vivo, poderiam contribuir para a ocorrência da atividade biológica. Sobretudo, considerando-se os resultados descritos por Suyenaga (2002), comprovando o mascaramento da atividade antiquimiotáxica de produtos metoxilados, devido à alta afinidade entre esse substituinte e a albumina presente no tampão utilizado no teste de quimiotaxia in vitro. Assim, para montanina, cuja molécula apresenta $\mathrm{C}_{2}$ metoxilação, talvez a atividade antiinflamatória deva ser melhor avaliada por meio da combinação do resultado já obtido para o ensaio in vitro, com dados provenientes da realização de métodos in vivo.

Porém, como estes resultados revelaram um grande potencial antimicrobiano para montanina, os quais corroboraram os dados farmacológicos e etnobotânicos disponíveis na literatura científica, os mesmos passam a ter importância se considerarmos que o desenvolvimento de novos antibióticos que inibam microrganismos cada vez mais resistentes, inclui a pesquisa com produtos naturais (Cole, 1994). Assim, estudos envolvendo alcalóides de Amarilidáceas e modificações estruturais podem constituir uma ferramenta para o desenvolvimento de novos produtos de interesse farmacêutico.

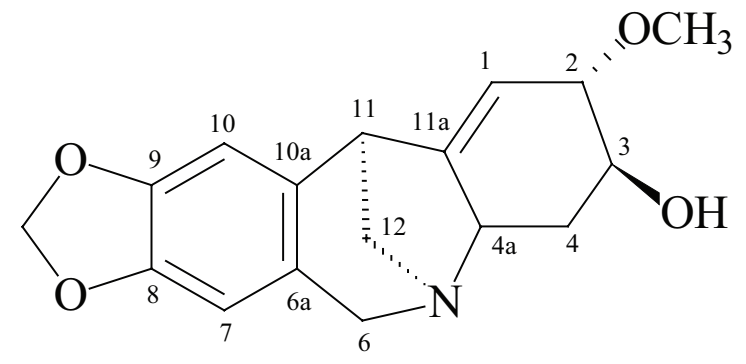

Figura 1. Estrutura química do alcalóide Montanina.

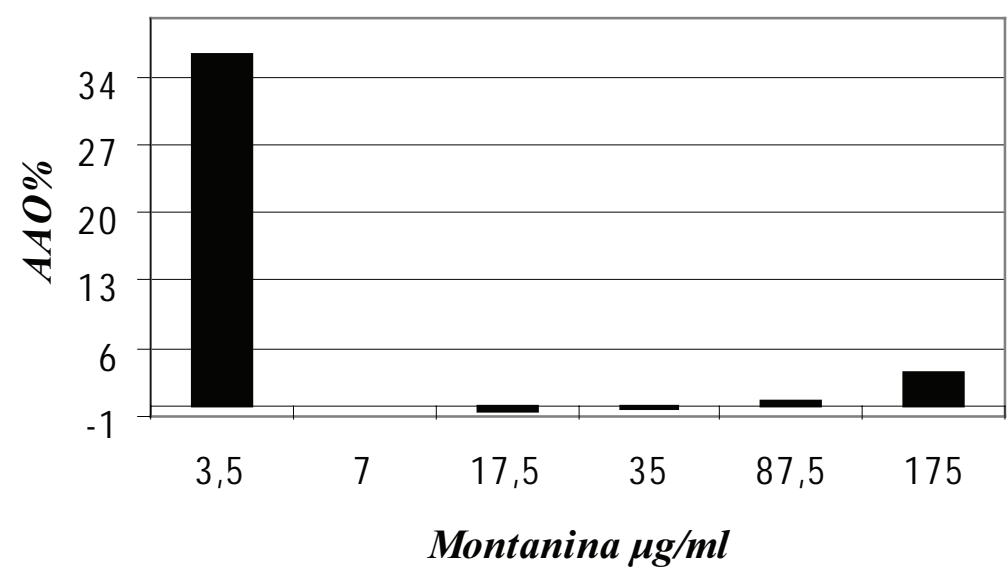

Gráfico 2. Representação gráfica da avaliação da atividade antioxidante da montanina cujos resultados não foram considerados significativos.

Tabela 1. Resultados da avaliação da atividade antiinflamatória da montanina através do método da Câmara de Boyden segundo a locomoção dos neutrófilos em suspensão.

\begin{tabular}{|l|c}
\hline Amostra & Distância Migrada $(\mu \mathrm{M})$ \\
\hline Controle & $133 \pm 1,4$ \\
Montanina & $126 \pm 0,9$ \\
\hline
\end{tabular}

Teste t de Student $\rho \leq 0,05$ 
Tabela 2. Concentração inibitória mínima de montanina frente aos microrganismos sensíveis.

\begin{tabular}{l|c}
\hline Microorganismo testado & Montanina $(\mu \mathrm{g})$ \\
\hline P. aeruginosa (ATCC 27853) & 20 \\
S. aureus (ATCC 6538) & 5 \\
S. epidermides (ATCC 12228) & 15 \\
E. coli (ATCC 25922) & 5 \\
S. cerevisiae (ATCC 2601) & 10 \\
\hline
\end{tabular}

\section{AGRADECIMENTOS}

À Capes e CNPq, pelo apoio financeiro.

\section{REFERÊNCIAS}

Agra MF, França PF, Barbosa-Filho JM 2007. Synopsis of the plants known as medicinal and poisonous in Northeast of Brazil. Rev Bras Farmacogn 17: 114-140.

Barbosa-Filho JM, Piuvezam MR, Moura MD, Silva MS, Lima KVB, Cunha EVL, Fechine IM, Takemura OS 2006a. Anti-inflammatory activity of alkaloids: A twentycentury review. Rev Bras Farmacogn 16: 109-139.

Barbosa-Filho JM, Medeiros KCP, Diniz MFFM, Batista LM, Athayde-Filho PF, Silva MS, Cunha EVL, Almeida JRGS, Quintans-Júnior LJ 2006b. Natural products inhibitors of the enzyme acetylcholinesterase. Rev Bras Farmacogn 16: 258-285.

Boyden S 1962. The chemotatic effect of mixtures of antibody and antigen on polymorphonuclear leukocytes. $J$ Exp Med 15: 453-466.

Bruneton J 2001. Farmacognosia, Fitoquímica, Plantas Medicináles, $2^{\mathrm{a}}$ edição, p.865-956, Ed. Acríbia S.A., Zaragoza, Espanha.

Cassels BK, Asencio M, Conget P, Speisky H, Videla LA, Lissi EA 1995. Structure-antioxidative activity relationships in benzylisoquinoline alkaloids. Pharm Res 31: 103107.

Castilhos T 2004. Investigação química em Habranthus erectus Ravenna e Rhodophiala bifida (Herb.) Traub (Amaryllidaceae) e Avaliação biológica in vitro do alcalóide montanina. Porto Alegre, 158p, Dissertação de Mestrado - Faculdade de Farmácia, Universidade Federal do Rio Grande do Sul.

Cole MD 1994. Key antifungal, antibacterial and anti-insect assays: Acritical review. Biochem Syst Ecol 22: 837856.

Cordell GA, Shamma M, Saxton JE, Smith GF 1989. Dictionary of alkaloids. In: Southon IW, Buckingham J (org.), Londres: Chapman and Hall Ltda, v. 1 e 2.

Dahlgren RMT 1985. The families of the monocotyledons: Structure, evolution and taxonomy, Berlim: SpringerVerlag, p.199-206.

Gabrielsen B, Monath TP, Huggins JW, Kefauver DF, Pettit GR, Groszek G, Hollingshead M, Kirsi JJ, Shannon WM, Schubert EM, DaRe J, Ugarkar B, Ussery
MA, Phelan MJ 1992. Antiviral (RNA) activity of selected Amaryllidaceae isoquinoline constituents and synthesis of related substances. J Nat Prod 55: 15691581.

Grove DC, Randall WA 1955. Assay methods of antibiotics: a laboratory manual, New York: Medical Encyclopedia Inc., p.80 (Antibiotics monographs, 02).

Hamburger MO, Cordell GA 1987. A direct bioautographic TLC assay for compounds possessing antibacterial activity. J Nat Prod 50: 19-22.

Hamburger MO, Hostettmann K 1991. Bioactivity in plants: the link between phytochemistry and medicine. Phytochemistry 30: 3864-3874

Harborne JB, Williams CA 2000. Advances in flavonoid research since 1992. Phytochemistry 55: 481-504.

Harvey A 1995. The pharmacology of galanthamine and its analogues. Pharmacol Ther 68: 113-128.

Hofmann Jr AE, Sebben C, Sobral MEG, Henriques AT, Zuanazzi JAS 2003. Alkaloids of Hippeastrum glaucescens (Martius) Herbert. Biochem Syst Ecol 31: 1455-1456.

Hofmann Jr AE, Sebben C, Montanha JA, Dutilh J, Sobral MEG, Henriques AT, Zuanazzi JAS 2004. Avaliação da atividade biológica e perfil cromatográfico de Hippeastrum glaucescens (Martius) Herbert. Rev Bras Farmacogn 14: 7-14.

Labraña J, Machocho AK, Kricsfalusy V, Brun R, Codina C, Viladomat F, Bastida J 2002. Alkaloids from Narcissus angustifolius subsp. trancarphaticus (Amaryllidaceae). Phytochemistry 60: 847-852.

Louw CAM, Regnier TJC, Korsten L 2002. Medicinal bulbous plants of South Africa and their traditional relevance in the control of infectious diseases. J Ethnopharmacol 82: 147-154.

Martinez LA, Rios JL, Paya M, Alcaraz MJ 1992. Inhibition of nonenzymic lipid peroxidation by benzylisoquinoline alkaloids. Free Radic Biol Med 12: 287-292.

Meerow AW, Snijan DA 1998. In: Kubitzki K. The families and genera of vascular plants. Berlin: Springer, p. 83110.

Mensor LL, Menezes FS, Leitão GG, Reis AS, Santos TC, Coube CS, Leitão SG 2001. Screening of Brazilian plant extracts for antioxidant activity by the use of DPPH free radical method. Phytother Res 15: 127130.

Rhee K, van de Meent M, Ingkaninan K, Verpoorte R 2001. 
Screening for acetylcholinesterase inhibitors from Amaryllidaceae using silica gel thin-layer chromatography in combination with bioactivity staining. J Chromatogr A 915: 217-223.

Rios JL, Recio MC, Villar A 1988. Screening methods for natural products with antimicrobial activity: A review of the literature. J Ethnopharmacol 23: 127-149.

Sebben C 2005. Investigação química em Hippeastrum breviflorum Herb. (Amaryllidaceae). Porto Alegre, 193p. Dissertação de Mestrado, Faculdade de Farmácia, Universidade Federal do Rio Grande do Sul.

Sener B, Orhan I, Satayavivad J 2003. Antimalarial activity screening of some alkaloids and the plant extracts from Amaryllidaceae. Phytother Res 17: 1220-1223.

Shale TL, Stirk WA, van Staden J 1999. Screening of medicinal plants used in Lesotho for anti-bacterial and antiinflammatory activity. J Ethnopharmacol 67: 347354.

Silva AFS 2005. Estudo químico e biológico de Hippeastrum vittatum (L Hér.) Herbert e Hippeastrum striatum (Lam.) Moore (Amaryllidaceae). Porto Alegre, 182p. Tese de Doutorado, Faculdade de Farmácia, Universidade Federal do Rio Grande do Sul.

Snedecor GW, Cochran WG 1974. Statistical methods applied to experiments in agriculture and biology. Iowa: The Iowa State University Press Ames.

Suyenaga ES 2002. Avaliação da atividade antiinflamatória de flavonóides por ensaios in vivo e in vitro. Porto Alegre, 238p. Tese de Doutorado, Faculdade de Farmácia, Universidade Federal do Rio Grande do Sul.

Traub HP 1953. The genera Rhodophiala Presl., Phycella Lindl. and Amaryllis L. Herbetia 9: 59-60.

Weniger B, Italiano L, Beck JP, Bastida J, Bergoñon S, Codina C, Lobstein A, Anton R 1995. Cytotoxic activity of Amaryllidaceae alkaloids. Planta Med 61: 77-79.

Wildmann WC, Brown CL 1968. Mass spectra of 5, 11bmethanomorphanthridine alkaloids. The structure of pancracine. J Am Chem Soc 90: 439-6446.

Zigmond SH, Hirsch JG 1973. Leukocyte locomotion and chemotaxis. New methods for evaluation and demostration of a cell-derived chemotatic factor. $J$ Exp Med 137: 387-410. 